\title{
Initial Phase of Infection with Tyzzer's Organism in Cultured Mouse Hepatocytes
}

\author{
By S. KAWAMURA, ${ }^{1 *}$ T. MITSUOKA ${ }^{1}$ AND K. FUJIWARA ${ }^{2}$ \\ ${ }^{1}$ Department of Biomedical Science, Faculty of Agriculture, The University of Tokyo, Yayoi, \\ Bunkyo-ku, Tokyo 113, Japan \\ ${ }^{2}$ Department of Veterinary Pathology II, Nihon University School of Veterinary Medicine, \\ Kameino, Fujisawa 252, Japan
}

(Received 19 September 1988; revised 14 March 1989; accepted 22 March 1989)

\begin{abstract}
The initial phase of infection with Tyzzer's organisms in cultured mouse hepatocytes was observed using indirect immunofluorescence (IF) and a plaque assay. The organisms adhered poorly to aldehyde- or acetone-fixed cells, but once adhered to host cells, whether methanolfixed or unfixed, they were not removed by methanol or acetone. By the IF as well as the plaque assay, both of which discriminated intra- and extracellularly located organisms, the number of cell-associated organisms increased linearly up to $3 \mathrm{~h}$ post-inoculation. The number of intracellular organisms increased rapidly in the first $1 \mathrm{~h}$, followed by linear increase at a much lower rate. Similar results were obtained by the plaque assay.
\end{abstract}

\section{INTRODUCTION}

Recently, host-parasite interactions have been studied at the cellular or molecular level in infections with bacteria which colonize mucosal surfaces, e.g. enterotoxigenic Escherichia coli, Vibrio cholerae, and Neisseria gonorrhoeae (Beachey, 1981), or with facultative intracellular parasites, e.g. Shigella flexneri, Salmonella typhimurium and enteroinvasive $E$. coli (Moulder, 1985). For obligate intracellular parasites, however, the early phase of infection has not been satisfactorily clarified, mainly because of the inaccessibility of the host or the microbial factors involved. Obligate intracellular infection at the cellular level consists of four phases, namely (i) adhesion (extracellular phase), (ii) entry (intracellular phase), (iii) multiplication, and (iv) release from host cells. Although specific receptors on host cells and entry mechanisms for several viruses have been determined at the molecular level, little information is available for obligate intracellular bacteria or protozoa, except for some rickettsiae, chlamydiae and malaria parasites (Moulder, 1985; Hadley et al., 1986).

The causative agent of Tyzzer's disease, which is characterized by liver necrosis and haemorrhagic enteritis (Tyzzer, 1917), is an obligate intracellular bacterium showing a strict tropism to highly differentiated cells in vivo, including hepatocytes (Fujiwara, 1978). We established an in vitro culture system for Tyzzer's organism using a primary monolayer culture of mouse hepatocytes (Kawamura et al., 1983 b). The present study was undertaken to study the initial phase of the infection in vitro, with the aid of indirect immunofluorescence (IF) techniques and a plaque assay, distinguishing between adhesion and entry of the organisms into the target cells.

\section{METHODS}

Animals. Female ICR mice were obtained from a commercial breeder (Charles River Japan, Atsugi, Japan). The breeder colony had been proved serologically to be free of the major murine pathogens: Tyzzer's organism,

Abbreviations: CM, complete medium; FITC, fluorescein isothiocyanate; TRITC, tetramethylrhodamine isothiocyanate; IF, indirect immunofluorescence; MEM, Eagle's minimal essential medium; p.i., postinoculation. 
Corynebacterium kutscheri, Bordetella bronchiseptica, Mycoplasma pulmonis, Salmonella typhimurium, murine hepatitis virus and Sendai virus (Fujiwara, 1971). Mice 4 to 5 weeks old were used for preparing infective materials and immune sera as well as for conducting the infectivity assay, while hepatocyte cultures were prepared from 8- to 14-week-old mice.

Hepatocyte cultures. Mouse hepatocytes for primary monolayer cultures were prepared by a modification of the methods of Seglen (1976) and Arnheiter (1980) as already described (Kawamura et al., 1983b). Primary monolayers of 24 to $30 \mathrm{~h}$ culture were obtained by seeding 1.5 or $3.5 \mathrm{ml}$ of cell suspension in culture medium (CM) containing $3.5 \times 10^{5}$ viable cells ml ${ }^{-1}$ in 35 or $60 \mathrm{~mm}$ plastic dishes, respectively. Cells were incubated at $37^{\circ} \mathrm{C}$ in a humid atmosphere of $5 \%(\mathrm{v} / \mathrm{v}) \mathrm{CO}_{2}$ in air. The $\mathrm{CM}$ consisted of Williams' medium $\mathrm{E}$ (Flow Laboratories) supplemented with $4 \mathrm{mU}$ insulin $\mathrm{ml}^{-1}$ and $1 \mu \mathrm{M}$-dexamethasone (Sigma). For establishing primary monolayer cultures, $\mathrm{CM}$ was further supplemented with heated calf serum at $5 \%(\mathrm{v} / \mathrm{v})$ and $50 \mu \mathrm{g}$ kanamycin $\mathrm{ml}^{-1}$. In some experiments, monolayers were fixed in the following fixatives for 5 to $10 \mathrm{~min}$ at $0{ }^{\circ} \mathrm{C}$ or room temperature $(22$ to $25^{\circ} \mathrm{C}$ ) before inoculation: methanol; ethanol; $10 \%(\mathrm{v} / \mathrm{v})$ formalin in $\mathrm{Ca}^{2+}$ - and $\mathrm{Mg}^{2+}$-free Dulbecco's phosphate-

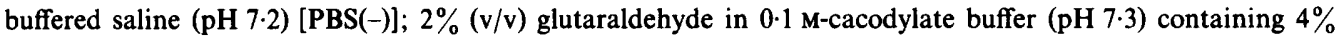
$(\mathrm{w} / \mathrm{v})$ sucrose; $2 \%(\mathrm{w} / \mathrm{v})$ paraformaldehyde in $0.1 \mathrm{M}$-phosphate buffer $(\mathrm{pH} 7.4)$ containing $8 \%(\mathrm{w} / \mathrm{v})$ sucrose; periodate/lysine/paraformaldehyde solution (McLean \& Nakane, 1974); and acetone. Fixed monolayers were immediately transferred to PBS(-) to avoid evaporation of fixatives and washed three times by stirring and changing the buffer. The fixed monolayers were returned to the culture dishes containing the medium, and inoculated as described below.

Organisms. The RT strain of rat-derived Tyzzer's organism (Yamada et al., 1969; Fujiwara et al., 1971) was used. A piece of heavily infected mouse liver which had been stored in liquid nitrogen was used to prepare inocula.

Antiserum. Mouse antiserum to the RT organisms was the same as described in the previous paper (Kawamura et al., 1983b).

Inoculation. The infected liver homogenate in kanamycin-free $\mathrm{CM}\left(37^{\circ} \mathrm{C}\right)$ was adjusted to 50 to 200 plaque forming units (p.f.u.) $\mathrm{ml}^{-1}$ and immediately $3.5 \mathrm{ml}$ each was inoculated onto monolayers in $60 \mathrm{~mm}$ culture dishes. The CM was changed once at 90 to $120 \mathrm{~min}$ post-inoculation (p.i.), and after further incubation for 36 to $42 \mathrm{~h}$ at $37^{\circ} \mathrm{C}$, the infected monolayers were removed from the culture dishes by a rubber policeman and homogenized together with culture supernate in a Teflon homogenizer at 1000 r.p.m. for $2 \mathrm{~min}$. After standing for $2 \mathrm{~min}$ to allow cell debris to sediment, the supernate of the homogenate was harvested and diluted with $\mathrm{CM}\left(37^{\circ} \mathrm{C}\right)$ before inoculation. In some experiments, only culture supernates of infected monolayers were harvested after repeated shaking of the dishes, and used as inocula. After the $\mathrm{CM}$ had been aspirated, monolayers on glass coverslips $(18 \times 24 \mathrm{~mm})$ were inoculated with freshly prepared inoculum and incubated at $37^{\circ} \mathrm{C}$. The inoculation process was completed within $30 \mathrm{~min}$ after harvesting the inoculum.

Immunofluorescence. The fixed and unfixed IF technique was similar to that described by Kihlstroem (1977) and Hale \& Bonventre (1979). Two groups of three to four inoculated monolayers were prepared for each experimental condition. After incubation for 60 to $180 \mathrm{~min}$ as indicated, the culture dishes were shaken repeatedly and the coverslips were washed three times with PBS(-) or Eagle's minimal essential medium (MEM) (Nissui Seiyaku, Tokyo, Japan) at room temperature $\left(22\right.$ to $\left.25^{\circ} \mathrm{C}\right)$. Then one group of infected monolayers was fixed in methanol for $10 \mathrm{~min}$ at room temperature, and then air-dried, while the other group remained unfixed. The monolayers were incubated with a 1 in 400 dilution of the anti-RT serum in MEM for $30 \mathrm{~min}$ at $4{ }^{\circ} \mathrm{C}$ or $37^{\circ} \mathrm{C}$, washed three times in PBS(-) or MEM for $3 \mathrm{~min}$ each, and then incubated for 20 to $30 \mathrm{~min}$ at room temperature with goat anti-mouse IgG (heavy and light chains specific) labelled with fluorescein isothiocyanate (FITC) or tetramethylrhodamine isothiocyanate (TRITC) (both from Cappel Laboratories) at 1 in 40 and 1 in 100 dilution in MEM, respectively. The monolayers were washed three times in PBS(-) or MEM for $5 \mathrm{~min}$ each, mounted in phosphate-buffered glycerin and examined by epifluorescence microscopy. The number of fluorescent bacteria was counted in 10 to 40 microscopic fields $(\times 200)$ per coverslip. The organisms on unfixed monolayers were considered as cell-surfaceassociated (extracellular), while those on fixed monolayers as total (extracellular plus intracellular). The mean number of intracellular bacteria was estimated as: (mean number of total bacteria) - (mean number of extracellular bacteria).

Double immunofluorescence. The method was described by Heesemann \& Laufs (1985) to discriminate directly between intracellularly and extracellularly located bacteria on a single monolayer. After washing, infected monolayers without prefixation were incubated with a dilution of anti-RT serum in MEM for $30 \mathrm{~min}$ at $4{ }^{\circ} \mathrm{C}$, washed three times in MEM each for $3 \mathrm{~min}$, and fixed in methanol for $10 \mathrm{~min}$ at room temperature. The fixed coverslips were air-dried and subsequently overlaid for 20 to $30 \mathrm{~min}$ at room temperature with TRITC-labelled anti-mouse IgG diluted in MEM to stain extracellularly adherent organisms. After washing in PBS(-), the monolayers were again incubated with anti-RT antibody for $30 \mathrm{~min}$ at room temperature, followed by washing and second staining with FITC-labelled anti-mouse IgG, which stained intracellular bacteria. The monolayers were washed in PBS(-), mounted and then examined by epifluorescence microscopy. In this method, cell-surfaceassociated (extracellular) organisms were observed by the TRITC filter system and total extracellular plus intra- 
cellular bacteria by the FITC system. The number of intracellularly located organisms was calculated as (number of total organisms) - (number of extracellular organisms) on each coverslip.

Plaque assay. The plaque assay technique (Kawamura et al., 1983a) was used to estimate kinetics of adhesion and entry of organisms to host cells and the results were compared to those obtained from the two IF procedures. Infected liver homogenate at an appropriate dilution was prepared at $37^{\circ} \mathrm{C}$ and inoculated onto hepatocyte monolayers in $35 \mathrm{~mm}$ culture dishes. After incubation for each period as indicated at $37^{\circ} \mathrm{C}$, three dishes were overlaid with agar medium immediately after aspirating the culture supernate. The plaques on these dishes were considered to be produced by total cell-associated bacteria, including reversibly adherent ones. The other six monolayers were washed three times in $\mathrm{CM}$ at room temperature with repeated crosswise shaking to remove reversibly adherent organisms and three of them were overlaid with agar medium. The remaining three were incubated with $\mathrm{CM}$ containing $100 \mu \mathrm{g}$ gentamicin $\mathrm{ml}^{-1}$ for $60 \mathrm{~min}$ at $37^{\circ} \mathrm{C}$ to kill extracellularly located organisms, followed by washing and the first overlay.

Reproducibility of results. The values shown in each Table or Figure were representative results of two to four independent experiments, except those in Table 1, which were means of two experiments in triplicate.

\section{RESULTS}

\section{Observations}

At 90 to 120 min p.i., fluorescence was positive only with cell-surface-associated organisms under epifluorescence microscopy (Fig. 1). In the case of infected monolayers fixed in methanol, IF revealed some organisms with peritrichous flagella (Fig. $1 a$ ), which were not clearly visible without methanol fixation (Fig. $1 b$ ). Some organisms on unfixed preparations were adherent aslant or perpendicular to host cells.

The micrographs in Fig. 2 show the same area of an infected monolayer after the double IF procedure, using two different filtering systems. Organisms located on the cell surface were visible under the TRITC filter system (Fig. 2a), while intracellular bacteria additionally appeared after FITC filtering (Fig. $2 b$ ). Under FITC microscopy, the extracellular organisms showed more distinct flagella than the intracellular ones.

\section{Effects of fixation}

As shown in Table 1, a significant decrease in number of bacteria adhering to hepatocytes was observed after treatment with various fixatives; methanol fixation had the least effect. There was a linear relationship between the number of organisms in inocula and those adhering to a defined area of either unfixed or methanol-fixed monolayers (Fig. 3).

Next, unfixed and methanol-fixed infected monolayers were incubated with antiserum and observed by IF with or without further fixation. Table 2 shows that the organisms remained adherent after fixation in methanol or in acetone. Organisms adherent to methanol-fixed hepatocytes were not removed by further treatment with methanol.

\section{Table 1. Adhesion of Tyzzer's organisms to fixed host cells}

Hepatocyte monolayers on coverslips were fixed in each fixative for $10 \mathrm{~min}$ at $4{ }^{\circ} \mathrm{C}$, and then incubated with RT organisms for $120 \mathrm{~min}$ at $37^{\circ} \mathrm{C}$.

\begin{tabular}{lc}
\multicolumn{1}{c}{ Fixation method } & $\begin{array}{c}\text { IF count } \\
(\%)\end{array}$ \\
Unfixed & $100^{*}$ \\
Methanol & $14 \cdot 3$ \\
Methanol (room temp., $22-25^{\circ} \mathrm{C}$ ) & $17 \cdot 3$ \\
Ethanol & $13 \cdot 0$ \\
$10 \%$ Formalin & $1 \cdot 8$ \\
$2 \%$ Glutaraldehyde & $1 \cdot 8$ \\
$2 \%$ Paraformaldehyde & 1.6 \\
Periodate/lysine/paraformaldehyde & $1 \cdot 5$ \\
Acetone & 0
\end{tabular}

* 1102 and 548 counts per 40 microscopic fields for the two experiments, respectively. 

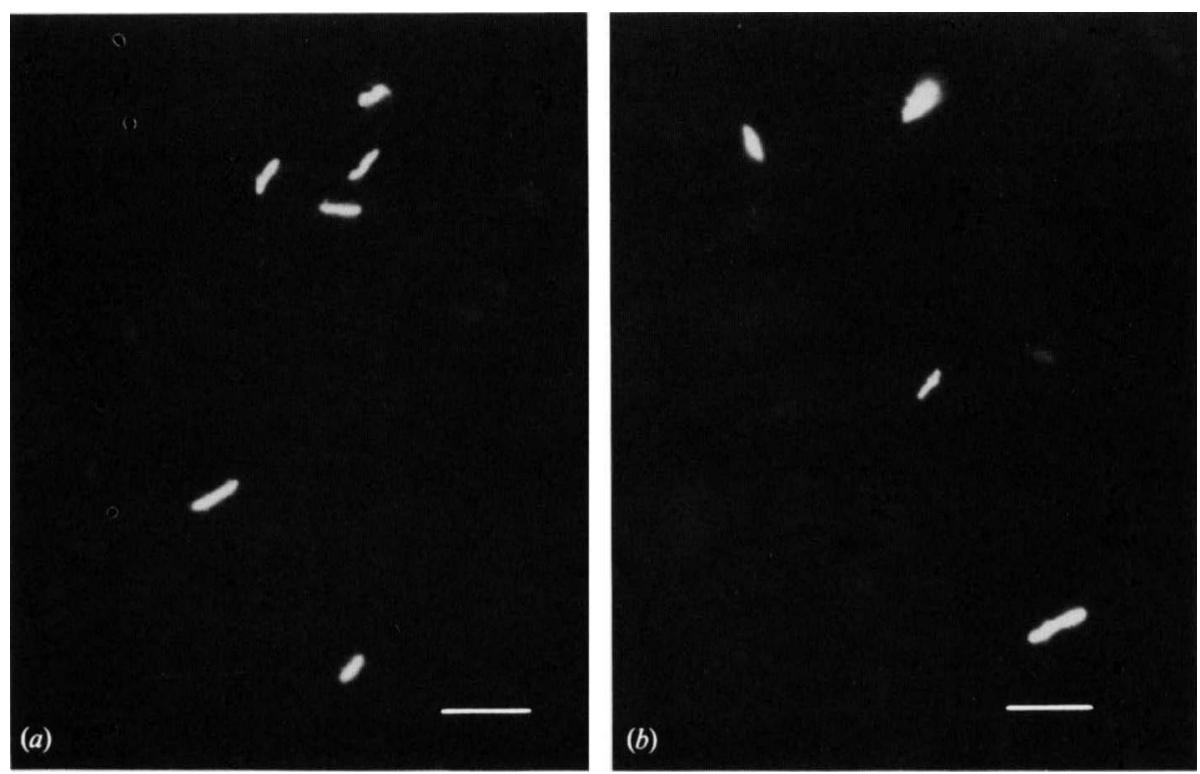

Fig. 1. Adhesion of RT strain of Tyzzer's organisms to cultured mouse hepatocytes at $2 \mathrm{~h}$ p.i. (a) Fixed and (b) unfixed IF using anti-RT antibody and TRITC-conjugate. Bars, $25 \mu \mathrm{m}$.
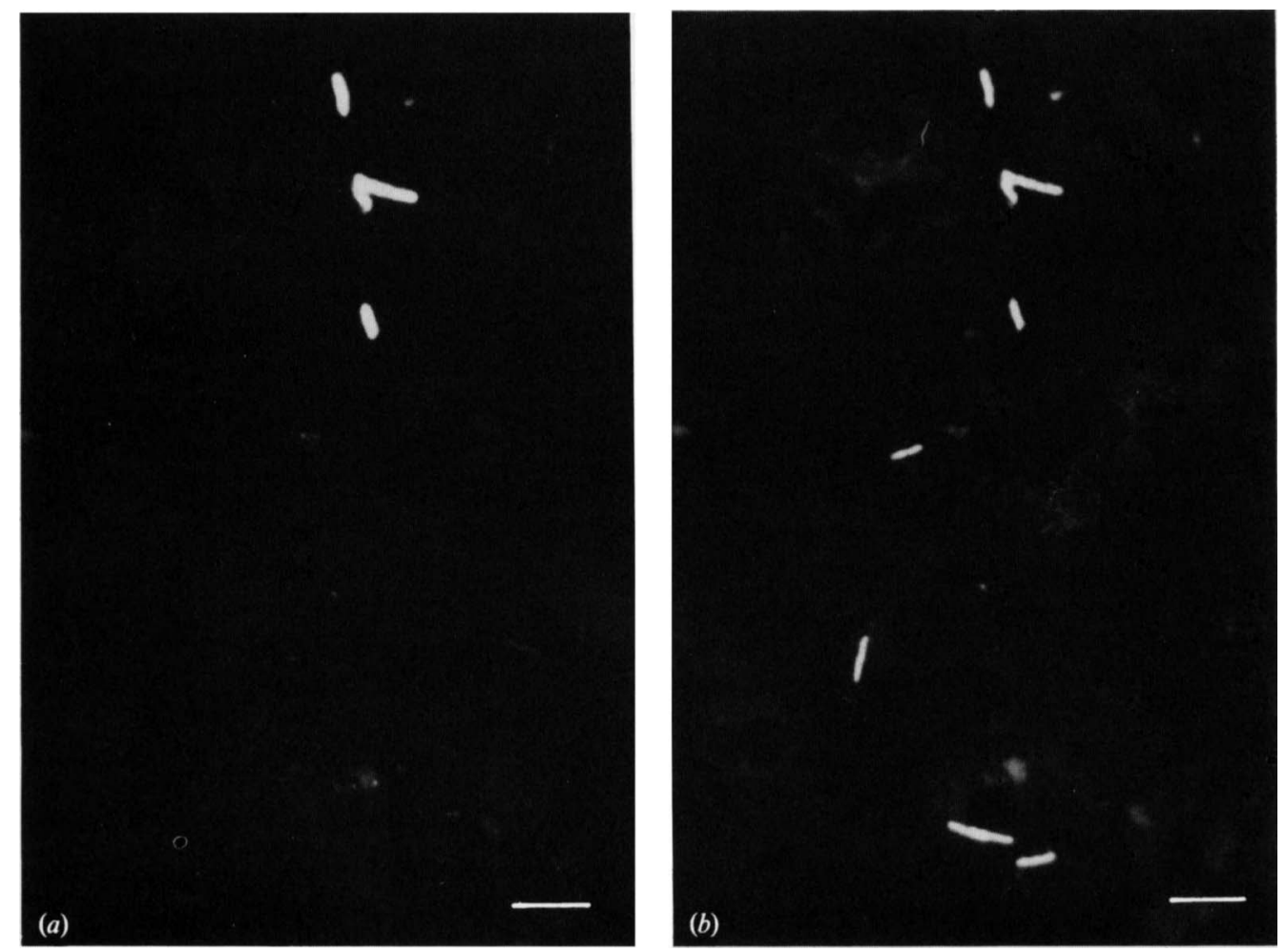

Fig. 2. Discrimination between extra- and intracellularly located Tyzzer's organisms in the same area of host cells. (a) Cell-surface-associated organisms under the TRITC filter system and $(b)$ total cellassociated organisms under the FITC filter system, using the double IF method. Bars, $25 \mu \mathrm{m}$. 


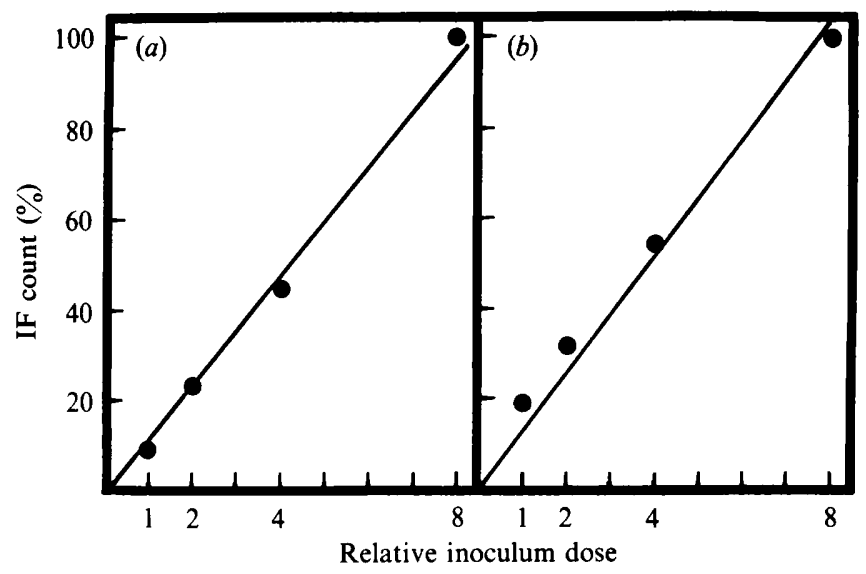

Fig. 3. Correlation between inoculum dilution and adherent organisms. Serial twofold dilutions of inocula were incubated with $(a)$ unfixed or $(b)$ methanol-fixed hepatocyte monolayers for $120 \mathrm{~min}$ at $37^{\circ} \mathrm{C}$. Mean IF counts of three coverslips were plotted. The absolute values corresponding to $100 \%$ are 278 and 470 counts per 40 microscopic fields for $(a)$ and $(b)$, respectively.

Table 2. Effect of methanol- or acetone-treatment on numbers of Tyzzer's organisms adherent to unfixed or fixed host cells

\begin{abstract}
Unfixed or methanol-fixed hepatocyte monolayers on coverslips were incubated with RT organisms for $120 \mathrm{~min}$ at $37^{\circ} \mathrm{C}$, treated with antiserum, and then fixed in each fixative for $10 \mathrm{~min}$ at room temperature.
\end{abstract}

$\begin{array}{lcc}\text { Treatment } & \text { Unfixed cells } & \text { Fixed cells } \\ \text { Unfixed } & 102 \pm 13 & 103 \pm 32 \\ \text { Methanol } & 103 \pm 12 & 107 \pm 24 \\ \text { Acetone } & 103 \pm 15 & \text { ND }\end{array}$

* Mean count of four coverslips \pm SD. ND, Not done.

\title{
Discrimination of adhesion and entry
}

The total number of cell-associated organisms increased almost linearly up to $3 \mathrm{~h}$ p.i. (Fig. $4 a$ ), suggesting that there was effectively no initial lag phase at any stage of infection. Thereafter reliable counting was impossible because of marked intracellular multiplication of organisms. The increase in number of extracellular bacteria during the first $3 \mathrm{~h}$ p.i. was much slower than that of total bacteria, and the number of intracellular bacteria, which was estimated from the total and cell-surface-associated numbers of bacteria, increased consistently. The percentage of intracellular organisms (percentage entry) increased rapidly during the first $1 \mathrm{~h}$, followed by a linear but slower increase (Fig. $4 \mathrm{~b}$ ). Entry of less than $30 \%$ at $90 \mathrm{~min}$ p.i. or later was considered to reflect failure in infection, and the data were discarded.

Fig. 5(a) shows that the results of the plaque assays were similar to those revealed by IF methods. The kinetics of percentage entry (Fig. $5 b$ ) also revealed a pattern almost identical to that shown in Fig. 4(b).

\section{DISCUSSION}

An in vitro cell culture system has many advantages for investigating the cytopathogenicity of micro-organisms. A relatively uniform population of host cells is available and can be infected under defined conditions. It is especially useful for obligate intracellular pathogens, which otherwise must be propagated in animal hosts. However, in studying an in vitro infection with 


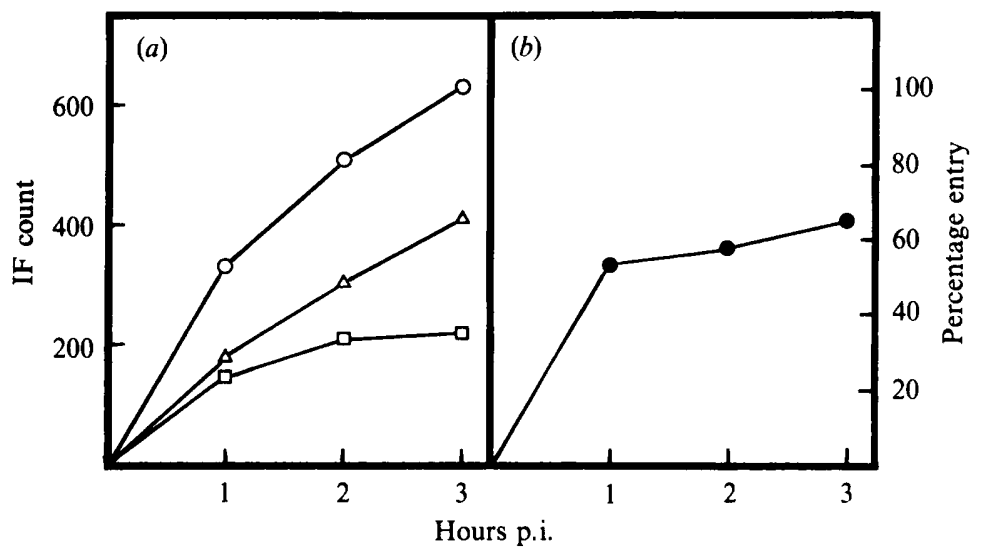

Fig. 4. Fixed and unfixed IF at the initial phase of infection with Tyzzer's organisms in cultured hepatocytes. (a) Mean counts of four coverslips stained by fixed $(O)(=$ total), or unfixed $(\square)(=$ extracellular) IF, and the difference between the two $(\triangle)$ (intracellular) were plotted. $(b)$ Percentage entry calculated from the data in $(a)$. Percentages of intracellular to total counts were plotted.

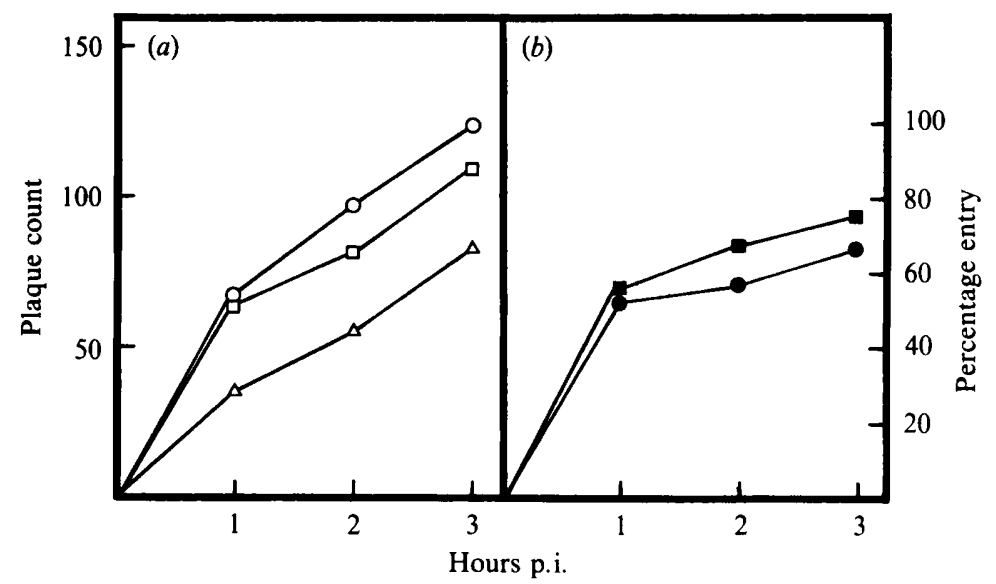

Fig. 5. Plaque assay at initial phase of infection with Tyzzer's organisms in cultured hepatocytes. (a) Mean plaque counts of three dishes which had been untreated $(O)$ (= reversible), washed $(\square)$ (=irreversible), or gentamicin-treated $\triangle)$ (=intracellular) were plotted. $(b)$ Percentage entry calculated from the data in $(a)$. Percentages of intracellular to reversible $(O)$ or to irreversible (D) adhesion were plotted.

intracellular micro-organisms, conventional light microscopy is not useful for accurate distinction of intracellular organisms from those associated with the cell surface. Also, transmission and scanning electron microscopy are not suitable for quantitative analysis.

The IF methods used in the present study were sufficient for accurate differentiation of the interaction between micro-organisms and host cells. The methods were based on inability of IgG to penetrate an intact cell membrane (Thomas et al., 1973), while passing through the membrane after treatment with fixatives. The method has been used for studies on neutrophil phagocytosis of gonococci (Dilworth et al., 1975) and on infection with facultative intracellular bacteria (Kihlstroem, 1977; Hale \& Bonventre, 1979). Heesemann \& Laufs (1985) reported a modification using a double IF staining procedure on a single preparation.

The two IF methods used in the present study were based upon the assumption that cellsurface-associated organisms were demonstrated only on unfixed preparations or by TRITC observation in the double IF technique, while the total of extracellular and intracellular organisms was shown on fixed preparations or by FITC observation in the double IF technique. 
Hale \& Bonventre (1979) noted that shigellae adherent to the surface of cultured enterocytes were totally removed by methanol treatment. However, Tyzzer's organisms adhering to cultured hepatocytes were not affected by treating with methanol or acetone, confirming the validity of the method employed in the present work.

With the in vitro system using fixed host cells, it is possible to study exclusively the adhesion phase of infection. As evidenced by Hatch et al. (1981), cell surfaces can be modified by fixatives, invalidating them as models for infections. In fact, the number of Tyzzer's organisms adherent to fixed hepatocytes was much smaller than that of organisms adherent to unfixed cells, suggesting change or degeneration, or masking of possible receptors for organisms by fixation. Nevertheless, methanol-fixed hepatocytes may provide a useful model for receptor studies and experiments using metabolic inhibitors which deteriorate unfixed cells. The fact that no organisms were adherent to aldehyde- or acetone-fixed cells suggests that the receptors, if any, were unstable in these solvents.

By the plaque assays, three categories of interaction between organisms and host cells were differentiated depending on degree of contact, that is, reversible and irreversible adhesions and intracellular localization. In the reversible adhesion, infective organisms remained in contact with host cells after removing culture medium and they could produce plaques. The irreversible adhesion was resistant to repeated shaking and washing of the monolayers. The intracellularly located organisms escaped the bactericidal action of gentamicin.

The discrimination of intra- and extracellular organisms by the IF methods was reliable because a similar pattern of the initial phase of infection was obtained in the kinetic studies using the plaque assay. The absence of a lag phase in the kinetics of internalization of organisms implied that invasion of host cells started immediately after inoculation. The data also demonstrated that some delays in entry might occur although entry consistently followed adhesion during the $3 \mathrm{~h}$ period p.i. The rate of entry slowed after $1 \mathrm{~h}$ p.i., suggesting that organisms were inactivated by prolonged incubation rather than there being some alteration of host cells, such as saturation in receptors or change in susceptibility to organisms, since the multiplicity of infection was very small in the present experiments.

In a preliminary experiment using homogenates of infected mouse liver as inocula, results were unsuccessful, because of a limited number of infective organisms and abundant cell debris which interfered with IF observation. Thus in the present study, the inocula for IF experiments were prepared from infected hepatocyte cultures; infection was successfully achieved with 1 in 10 or 1 in 20 dilutions of homogenates of infected hepatocyte monolayers.

This study was supported in part by a Grant-in-Aid for Science Research no. 61780055 from the Ministry of Education, Science and Culture, Japan.

\section{REFERENCES}

ARnheIter, H. (1980). Primary monolayer culture of adult mouse hepatocytes - a model for the study of hepatotropic viruses. Archives of Virology 63, 11-22.

BEACHEY, E. H. (1981). Bacterial adherence: adhesinreceptor interactions mediating the attachment of bacteria to mucosal surfaces. Journal of Infectious Diseases 143, 325-345.

Dilworth, J. A., Hendley, J. O. \& Mandell, G. L. (1975). Attachment and ingestion of gonococci by human neutrophils. Infection and Immunity 11, 512-516.

FujIWARA, K. (1971). Problems in checking inapparent infections in laboratory mouse colonies: an attempt at serological checking by anamnestic response. In Defining the Laboratory Animal, pp. 7792. Edited by H. A. Schneider. Washington, DC: National Academy of Sciences.

FujiWARA, K. (1978). Tyzzer's Disease. Japanese Journal of Experimental Medicine 48, 467-480.
Fujiwara, K., Yamada, A., Ogawa, H. \& Oshima, Y. (1971). Comparative studies on the Tyzzer's organisms from rats and mice. Japanese Journal of Experimental Medicine 41, 125-133.

Hadley, T. J., Klotz, F. W. \& Miller, L. M. (1986). Invasion of erythrocytes by malaria parasites: a cellular and molecular overview. Annual Review of Microbiology 40, 451-477.

Hale, T. L. \& Bonventre, P. F. (1979). Shigella infection of Henle intestinal epithelial cells: role of the bacterium. Infection and Immunity 24, 879886.

Hatch, T. P., Vance, D. W. \& Al-Hossainy, E. (1981). Attachment of Chlamydia psittaci to formaldehyde-fixed and unfixed $\mathrm{L}$ cells. Journal of General Microbiology 125, 273-283.

HeEsemanN, J. \& Laufs, R. (1985). Double immunofluorescence microscopic technique for accurate differentiation of extracellularly and intracellularly 
located bacteria in cell culture. Journal of Clinical Microbiology 22, 168-175.

Kawamura, S., Taguchi, F. \& Fujiwara, K. (1983a). Plaque formation by Tyzzer's organism in primary monolayer culture of mouse hepatocytes. Microbiology and Immunology 27, 415-424.

Kawamura, S., Taguchi, F., Ishida, T., Nakayama, M. \& Fujiwara, K. $(1983 b)$. Growth of Tyzzer's organism in primary monolayer cultures of adult mouse hepatocytes. Journal of General Microbiology 129, 277-283.

KinLSTROEM, E. (1977). Infection of HeLa cells with Salmonella typhimurium 395 MS and MR10 bacteria. Infection and Immunity 17, 290-295.

Mclean, I. W. \& Nakane, P. K. (1974). Periodatelysine-paraformaldehyde. A new fixative for immunoelectron microscopy. Journal of Histochemistry and Cytochemistry 22, 1077-1083.
Moulder, J. W. (1985). Comparative biology of intracellular parasitism. Microbiological Reviews 49, 298-337.

SEgLen, P. O. (1976). Preparation of isolated rat liver cells. Methods in Cell Biology 13, 29-83.

Thomas, D. W., Hill, J. C. \& Tyeryar, F. J. (1973). Interaction of gonococci with phagocytic leukocytes from men and mice. Infection and Immunity 8, 98-104.

TyzzER, E. E. (1917). A fatal disease of the Japanese waltzing mouse caused by a spore-bearing bacillus (Bacillus piliformis n.sp.). Journal of Medical Research 37, 307-338.

Yamada, A., Osada, Y., Takayama, S., Akimoto, T., Ogawa, H., Oshima, Y. \& Fujiwara, K. (1969). Tyzzer's disease syndrome in laboratory rats treated with adrenocorticotropic hormone. Japanese Journal of Experimental Medicine 39, 505-518. 